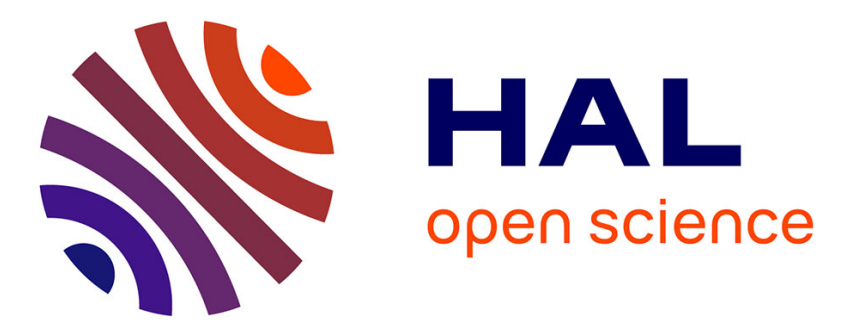

\title{
Focusing Dynamics of High-Energy Density, Laser-Driven Ion Beams
}

S. N Chen, E d'Humières, E. Lefebvre, L. Romagnani, T. Toncian, P. Antici, P. Audebert, E. Brambrink, C. A Cecchetti, T. Kudyakov, et al.

\section{- To cite this version:}

S. N Chen, E d'Humières, E. Lefebvre, L. Romagnani, T. Toncian, et al.. Focusing Dynamics of High-Energy Density, Laser-Driven Ion Beams. Physical Review Letters, 2012, 108 (5), pp.055001. 10.1103/PhysRevLett.108.055001 . hal-03321061

\section{HAL Id: hal-03321061 https://hal.science/hal-03321061}

Submitted on 17 Aug 2021

HAL is a multi-disciplinary open access archive for the deposit and dissemination of scientific research documents, whether they are published or not. The documents may come from teaching and research institutions in France or abroad, or from public or private research centers.
L'archive ouverte pluridisciplinaire HAL, est destinée au dépôt et à la diffusion de documents scientifiques de niveau recherche, publiés ou non, émanant des établissements d'enseignement et de recherche français ou étrangers, des laboratoires publics ou privés. 


\section{Focusing dynamics of high-energy density, laser-driven ion beams}

S. N. Chen ${ }^{1,2}$, E. d'Humières ${ }^{3,4}$, E. Lefebvre ${ }^{5}$, L. Romagnani ${ }^{1}$, T. Toncian ${ }^{6}$, P. Antici $^{1}$, P. Audebert ${ }^{1}$, E. Brambrink $^{1}$, C. A. Cecchetti ${ }^{7}$, T. Kudyakov ${ }^{6}$, A. Pipahl ${ }^{6}$, Y. Sentoku ${ }^{3}$, M. Borghesi ${ }^{7}$, O. Willi ${ }^{6}$, J. $^{2}$ Fuchs ${ }^{1, *}$

${ }^{1}$ LULI, École Polytechnique, CNRS, CEA, UPMC, route de Saclay, 91128 Palaiseau, France

${ }^{2}$ Lawrence Livermore National Laboratory, Livermore, CA 94551, USA

${ }^{3}$ Nevada Terawatt Facility, MS-372, University of Nevada, Reno, Nevada 89557, USA

${ }^{4}$ Université de Bordeaux, CNRS, CEA, Centre Lasers Intenses et Applications, 33400, Talence, France ${ }^{5}$ CEA, DAM, DIF, 91297 Arpajon, France

${ }^{6}$ Heinrich Heine Universität Düsseldorf, D-40225 Düsseldorf, Germany

${ }^{7}$ School of Mathematics and Physics, The Queen's University Belfast, Belfast BT7 1NN, Northern Ireland, United Kingdom

The dynamics of the focusing of laser driven ion beams produced from concave solid targets was studied. Most of the ion beam energy is observed to converge at the center of the cylindrical targets with a spot diameter of $30 \mu \mathrm{m}$, which can be very beneficial for applications requiring high beam energy densities. Also, unbalanced laser irradiation does not compromise the focusability of the beam. However, significant filamentation occurs during the focusing, potentially limiting the localization of the energy deposition region by these beams at focus. These effects could impact the applicability of such high-energy density beams for applications, e.g. in proton-driven Fast Ignition.

*julien.fuchs@polytechnique.fr 
The ability to tightly focus, i.e. over tens of microns, dense $\left(>10^{10}\right.$ particles), short (with duration $\sim$ ps) bunches of positively charged particles is far beyond the possibility of present particle accelerators. The increase of the particle density of these beams would significantly improve the efficiency and prospects of a number of important applications. High energy density proton beams would allow, for example, igniting pre-assembled Inertial Confinement Fusion (ICF) targets, also known as proton Fast Ignition (PFI) [1], or enable ultrafast heating, above $\mathrm{keV}$, of dense materials to explore their properties [2]. High energy density positron beams would allow enhancing the rate of antimatter creation in the laboratory [3], opening up investigations of many fundamental laws of nature.

These positively charged beams, broadband or monoenergetic, can nowadays be produced with a high particle number and over a short duration using ultra-intense, short pulse lasers interacting with solids targets $[4,5]$. Proton beams are electrostatically accelerated by $\mathrm{MeV}$ hot electrons. These electrons are generated at the laser-solid interaction surface. After crossing the target, they induce a Debye sheath on the target rear surface. The quasi-electrostatic fields in the sheath then accelerate surface ions [6,7]. Acceleration of positrons, which are produced in the bulk through nuclear processes, when they exit the solid target have also been reported [5]. When using flat targets as sources, the ion beam is divergent, 025 degrees depending on proton energy $[8,9,10]$, since the expanding sheath field front on the target rearside is Gaussian in shape [11,12]. Such divergence can however be compensated by curving the back surface of the target, so that the accelerated proton beam will converge $[4,13,14,15]$. This points to the necessity of understanding and optimizing the dynamics of the focusing sheath in order to achieve tight focusing, as necessary for the above-mentioned applications.

In this Letter, we study the ion beam focusing dynamics through temporally and spatially resolved measurements, with picosecond and micrometer resolution, of the shape of the sheath field from a curved target, irradiated by a high-intensity short pulse laser. These are coupled to numerical simulations performed using the 2-D Particle-In-Cell (PIC) codes CALDER [16] and PICLS [17], and a particle transport code, CMC [18]. The main results are as follows: (i) a convergence of the ion beam accelerated from the inner surface of a $800 \mu \mathrm{m}$ diameter cylinder is observed to $\sim 30 \mu \mathrm{m}$ diameter, (ii) significant filamentation however occurs in the converging plasma, (iii) the location of the focus is a function of proton energy, although most of the protons focus at the geometric target center, and (iv) the exact location of laser illumination on the curved target does not adversely affect the ability to focus the sheath-accelerated ion beam, although it modifies the directionality of the ion beam.

The experiment was carried out using the $100 \mathrm{TW}$ laser at the Laboratoire pour l'Utilisation des Lasers Intenses (LULI) operating in the chirped pulse amplification (CPA) mode. To diagnose the sheath 
field structure, we used the now established technique of transverse proton radiography, which can uniquely resolve, in space and time, quasi-static fields in dense plasmas [19]. Radiography using laserdriven proton beam is unique in that it is able to probe both electric and magnetic fields in dense plasmas with high temporal and spatial resolution, thanks to the low emittance of the beam and the short emission duration [20]. Studying the field structures in a 3-D curved target, such as a hemisphere, is very difficult, if not impossible. Therefore, by using a simpler curved geometry, such as a half cylinder, we can study the fields in 2-D. The experimental set-up is shown in Figure 1. The wavelength of the laser light was $1.057 \mu \mathrm{m}$ and the pulse duration was $\tau=320 \mathrm{fs}$, as measured after compression and before focusing. Two short pulse beams were used. The first short pulse beam (B1), with $1 \mathrm{~J}$ in energy, $11 \mu \mathrm{m}$ spot diameter, and intensity of $2.5 \times 10^{18} \mathrm{~W} / \mathrm{cm}^{2}$ on target, was used to irradiate aluminum half cylinders, $670 \mu \mathrm{m}$ long, having $50 \mu \mathrm{m}$ thick walls and $800 \mu \mathrm{m}$ diameter, producing protons with energy up to $1 \mathrm{MeV}$. The second independently compressed short pulse beam (B2), with $7 \mathrm{~J}$ in energy, $7 \mu \mathrm{m}$ spot diameter, and intensity of $4.4 \times 10^{19} \mathrm{~W} / \mathrm{cm}^{2}$, irradiated a $10 \mu \mathrm{m}$ thick gold foil to produce protons with energy up to $15 \mathrm{MeV}$, which were used for proton radiography. This technique inherently takes advantage of proton time-offlight [21] such that the highest energy protons will probe the cylinder at the beginning of the interaction and the slower protons will probe later in time. Therefore, multiframe snapshots of the fields' evolution can be captured on a single shot.

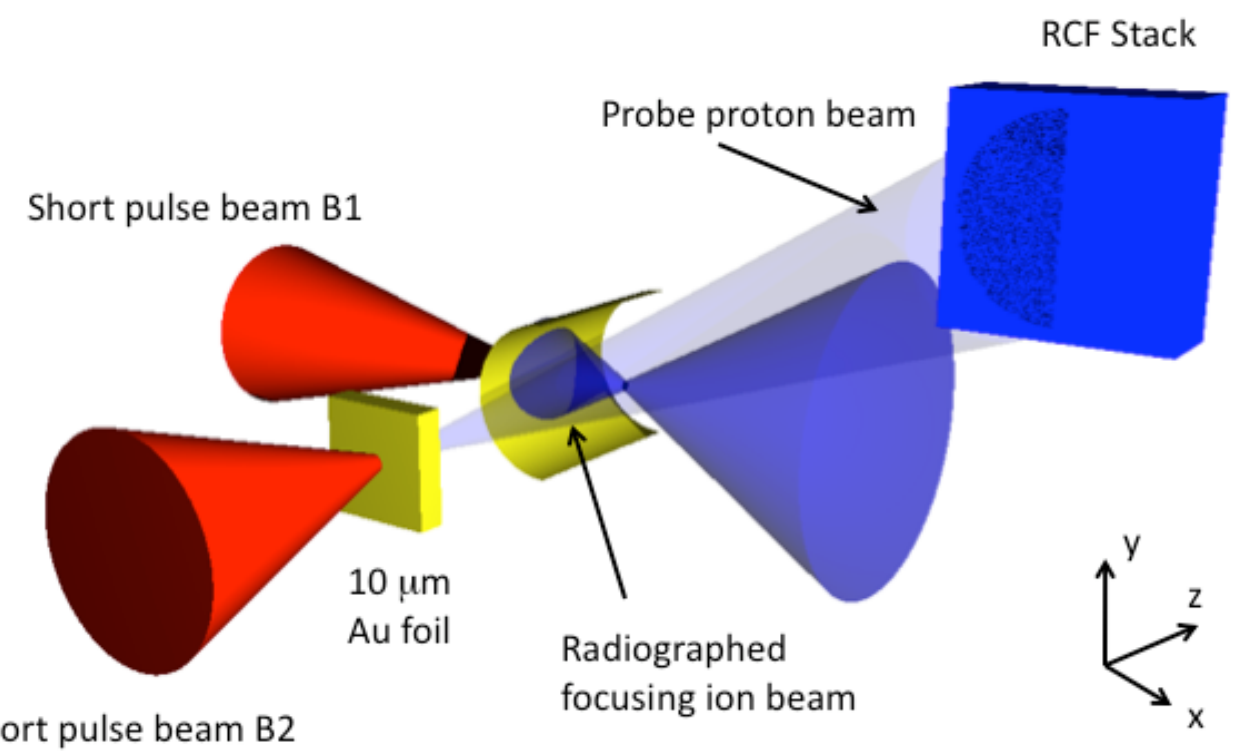

Figure 1: Experimental setup (see text). 
The detector was a stack of radiochromic film (RCF) where the deflected protons will deposit most of their energy at a depth within the stack according to their Bragg curve. The stack was composed of four pieces of Gafchromic HD-810 film followed by MD-55 films with a $13 \mu \mathrm{m} \mathrm{Al}$ foil filter at the very front to block unwanted radiation. Distance from the Au foil to the B1 irradiation point on the cylinder is 3 $\mathrm{mm}$ and from the cylinder to the RCF is $25 \mathrm{~mm}$. On some shots, a copper mesh was placed $2 \mathrm{~mm}$ from the $\mathrm{Au}$ foil, i.e. before the half cylinder target, to imprint a grid pattern onto the proton probe beam to explicitly show the magnitude and direction of the fields [20].

Side-on experimental radiographs of the inner surface of the half cylinder target are shown in Fig. 2a$d$ and all times are normalized to $t_{\text {arrival }}$ at which the highest energy protons arrive at the focus point. The dark regions, indicative of proton pile-up, outline the expanding sheath field created by the hot electrons [11]. The first radiograph of the series is taken at $2 \mathrm{ps}$ after irradiation of the cylinder, a time at which the cloud of hot electrons produced during the laser interaction has already surrounded the entire half cylinder uniformly (not shown). In Fig. 2a, at $\mathrm{t}=4 \mathrm{ps}$, we can see that in addition to this general sheath front, at the center of the half cylinder, there is another spherically shaped front which is expanding faster. As observed in previous experiments, this front is produced by the $\sim \mathrm{MeV}$ hot electrons that accelerate the highest energy ions [7,11], with the lower energy protons being accelerated behind the front by lower amplitude fields $[4,6]$. At $\mathrm{t}=11 \mathrm{ps}$, the spherical expansion front disappears and short filaments appear inside the expanding plasma, which is consistent with what has been simulated by PIC codes [22]. This can be attributed to either electromagnetic or electro-thermal instabilities [23]. As time progresses, the filaments grow and elongate. We have previously observed [8] that such a filamented sheath will give rise to a filamented proton beam. Therefore, this will impair the homogeneity of the final produced beam. However, in our observations, we can also use these filaments as a fiducial which indicates the direction of plasma flow since the filaments are produced in the direction of the hot electron flow. Thus, despite the filamentation, we can witness that the plasma begins to converge after $10 \mathrm{ps}$.

At $t=27 \mathrm{ps}$, the filaments reach a crossing point which defines $t_{\text {arrival }}$. The convergence is clearest at $\mathrm{t} / \mathrm{t}_{\text {arrival }}=1.77$ and the image is shown in Fig. 2c. It should be noted that in the radiographs at $\mathrm{t} / \mathrm{t}_{\text {arrival }}=$ 1.77, Fig. 2c-d, there appears to be two sets of filaments: a shorter set close to the cylinder wall and a longer set within the expansion region. The shorter set is likely due to filamentation in the general sheath field, which extends over the entire target, but remains close to the target surface. The longer set is only produced close to the laser interaction area and is associated with the progressing proton cloud and its localized focusing. Since the radiographs integrate over the $670 \mu \mathrm{m}$ length of the target, both sets are captured on the radiograph. Note that filamentation could not be observed in the first experiment 
reporting proton focusing [13], due to limited spatial resolution and because the focusing was observed indirectly by measuring the heating of a secondary target placed at the ion beam focus. The focus of the central filaments and of the overall beam envelope is $370 \mu \mathrm{m}$ away from the inner surface of the cylinder, which is consistent with the radius of curvature of the cylinder, and has a spot size of $\sim 30 \mu \mathrm{m}$ diameter. This gives a $D / r=0.93$ with $D$ being the location of focus relative to the target back surface and $r$ the radius of curvature of the target. In projecting the shorter filaments, corresponding to lower energy protons accelerated far from the laser irradiation location, they too come to a cross point, but this time at $400 \mu \mathrm{m}$ from the back surface and the projections make a much larger spot size of $100 \mu \mathrm{m}$, which is consistent with previous data showing that lower energy protons have a larger divergence $[9,10]$. We can estimate the proton current density at focus. For this, we take our laser parameters, a spread of proton energies from $500 \mathrm{keV}$ to $1 \mathrm{MeV}$, a proton conversion efficiency of $1 \%$ of the laser energy [24], and a spot area of $9 \times 10^{-6} \mathrm{~cm}^{2}$. The difference in time of arrival at focus is $11 \mathrm{ps}$ which gives a current density of $1 \times 10^{8} \mathrm{~A} \cdot \mathrm{cm}^{-2}$.

The radiographs of the half cylinders shown in Fig 2c-d are for early times. The radiographs in Fig 2e$\mathrm{f}$ are shown using curved targets, made from a large foil moulded onto a rod. They show the sheath field structure later in time. Both the filaments and caustic features also come to a crossing point near the radius of curvature and then diverge after the focus point as is predicted in the simulation shown in Fig. $3 \mathrm{c}$ (detailed below). Note that the structures appearing in Fig 2e-f are not necessarily in a one-to-one correspondence to the filaments within the converging ion beam since they represent modulations induced on the probing proton beam passing through the plasma and projected far away on the film. Nevertheless, the fact that such structures appear in the probing proton beam implies that the converging proton beam is indeed inhomogeneous.

We used CALDER and CMC codes to simulate, in 2-D geometry, laser-cylinder interaction, proton acceleration and focusing. Here, the CALDER code is used to calculate the laser-target interaction, electromagnetic fields, and particle positions up to $1.2 \mathrm{ps}$ after the interaction; then information on protons velocity and direction are used in $\mathrm{CMC}$ to trace particle trajectories for longer times than what can reasonably be done in standard PIC codes, until and after the ion beam reaches its focus. 

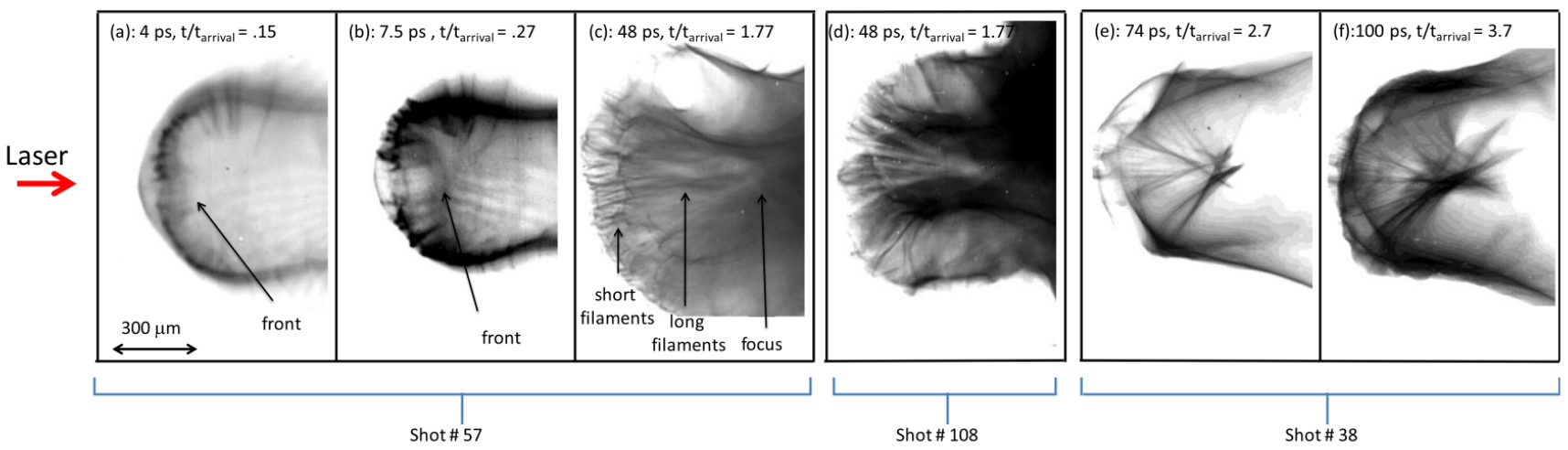

Figure 2: (a-d) Selected proton deflectograms of only the interior of the half cylinder target in time up to 48 ps. (e-f) Proton deflectograms of the interior of bent foils later in time. The contrast of the images was optimized to show the structures in the radiograph. The laser irradiates the half cylinder from the left side (the interaction side of the target is not shown). All times are normalized to $t_{\text {arrival }}=27 \mathrm{ps}$.

In the simulation, the half cylinder has a radius of curvature of $80 \mu \mathrm{m}$ ( 5 times smaller than the experimental target) and the laser intensity is $10^{18} \mathrm{~W} / \mathrm{cm}^{2}$, accelerating protons to a maximum energy of several MeVs, as we see experimentally. The convergence time is $t_{\text {arrival }}=5.7 \mathrm{ps,} \mathrm{i.e.} 5$ times smaller than the experimental $t_{\text {arrival }}$, which is consistent with the experimental results of a reduced scale target. The evolution of the proton beam in the simulation is consistent with the measured data, such that early in time, the protons, accelerated off the back surface of the target, also exhibit a convex shape in the central region (see Fig 2b) and later on transformed into a focused beam (see Fig 3a compared to 3b). Once protons travel beyond the focus point, they tend to diverge again as shown in Fig. 3c. Furthermore, we found that the focus is chromatic such that the higher energy protons focus farther than the lower energy protons. Hence, the location of the proton focus changes in time and extends over $110 \mu \mathrm{m}$ as shown in Fig. 4.b. The highest energy protons originate only near the laser interaction region and converge at a distance $110 \mu \mathrm{m}$ farther than the geometrical focus, giving a ratio $D / r=2.4$, which is consistent with previous findings [25]. However, the bulk of the protons are lower in energy $(\sim 1 \mathrm{MeV})$ and they originate from the entire surface of the sheath and converge at its geometrical center, giving a ratio $D / r=$ 1 , which is consistent with the behaviour of the short filaments in the experimental images.

Finally, we performed simulations with higher laser intensity in order to produce protons which have experiments higher than in the experiments, but close to the one requited for fast ignition [1]. The proton beam characteristics for laser intensity of $8 \times 10^{18} \mathrm{~W} / \mathrm{cm}^{2}$ is shown in Fig. $4 \mathrm{c} \& 4 \mathrm{~d}$. The higher intensity 
increased the proton spectrum up to $25 \mathrm{MeV}$, reduced the beam waist at the focus location for lower energy protons, and reduced the spread of distances that the protons focus to as a function of energy, as is beneficial for fast ignition. It also suggests that with even higher laser intensities, focusing of the proton beam will improve even more.

Beyond determining the dynamics of the focusing, we also tested the effect of unbalanced irradiation onto the half-cylinder target. Indeed, in future large-scale ICF facilities (HiPER [26], NIF-ARC [27]), short pulse beams will use several "beamlets" to construct the overall laser beam that will impinge onto the target. In view of PFI applications, where these beamlets will irradiate an hemispherical shell to produce the igniting proton beam [28], there is a clear interest in testing how ion beam focusability can be affected by an unbalanced distribution of intensity on the shell.
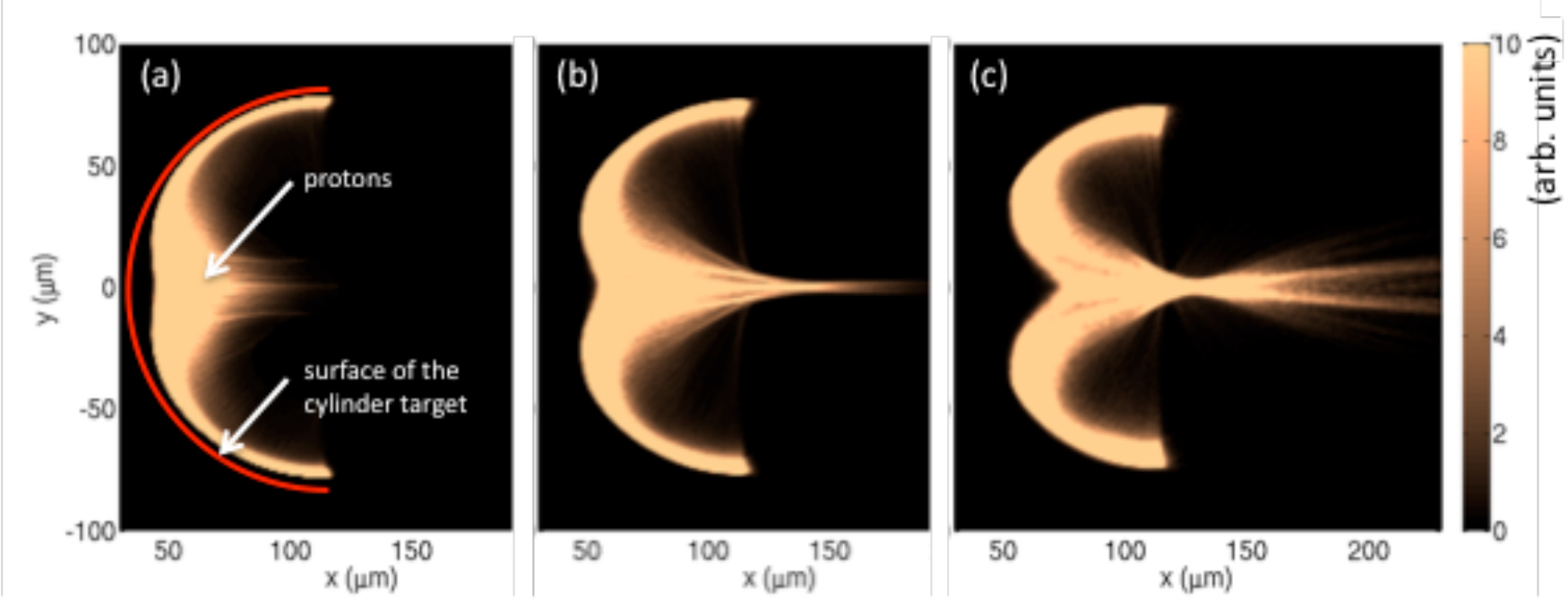

Figure 3: CMC simulation results showing proton position in the interior of the half cylinder target at (a) $\mathrm{t} / \mathrm{t}_{\text {arrival }}=.47$, (b) $\mathrm{t} / \mathrm{t}_{\text {arrival }}=1$, (c) $\mathrm{t} / \mathrm{t}_{\text {arrival }}=2$. All times are normalized to $\mathrm{t}_{\text {arrival }}=5.7 \mathrm{ps}$.

Here we tested this by taking one shot where the short pulse laser B1 was aimed slightly beneath the axis of the half cylinder. In Fig 5a \& 5b, proton radiographs at two different times for the same shot are shown. It is obvious that off-axis irradiation prevents formation of a uniform sheath field, with a larger field observed at the edge of the cylinder closest to where the laser impacted. 

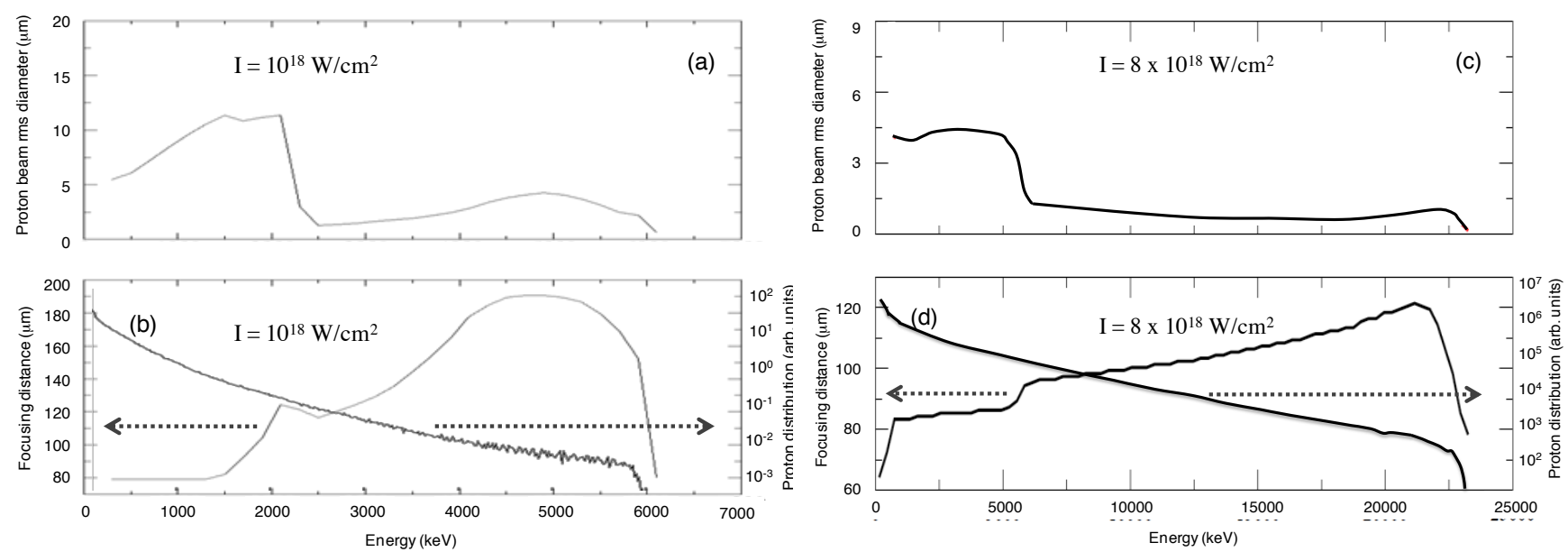

Figure 4: Simulation results from CALDER and CMC. (a \& b) Proton beam waist and focusing location as a function of proton energy when laser intensity is $10^{18} \mathrm{~W} / \mathrm{cm}^{2}$. (b) Same, but using laser intensity of 8 $\mathrm{x} 10^{18} \mathrm{~W} / \mathrm{cm}^{2}$.

We simulated this scenario using a half cylinder with a radius of $42 \mu \mathrm{m}$ (10 times smaller than the experimental target) and thickness of $5 \mu \mathrm{m}$ using the code PICLS. The simulation box was $92 \times 92 \mu \mathrm{m}^{2}$, the laser intensity of $3 \times 10^{18} \mathrm{~W} / \mathrm{cm}^{2}$ with a pulse duration of $175 \mathrm{fs}$ and FWHM of $6 \mu \mathrm{m}$. Fig. $5 \mathrm{c}-\mathrm{d}$ show the spatial position of the hot electrons with energy of 100 to $500 \mathrm{keV}$ at different times (normalized to $t_{\text {arrival }}=1.5 \mathrm{ps}$ ). As expected, the edge closest to where the laser hit the target has a higher concentration of hot electrons than the ends of the target. This assymmetry is maintained even later in time as the sheath expands into vacuum due to the fact that electrons take more time to transit to the far distant edge and to come back to their initial location than they do relative to the closer edge. The influence of such transverse electron refluxing dynamics [29] leads to an uneven development of the sheath field. However, when looking at the accelerated protons as shown in Fig 5e-f, this is not detrimental to proton beam focusing, but only changes the directionality of the focused beam itself. It is clear from the contours in the proton beam that they do still converge to a point. In more general terms, balancing of the laser beamlets that would compose the single laser beam on target used for PFI would thus not be important in terms of the ability to focus the ion beam. 


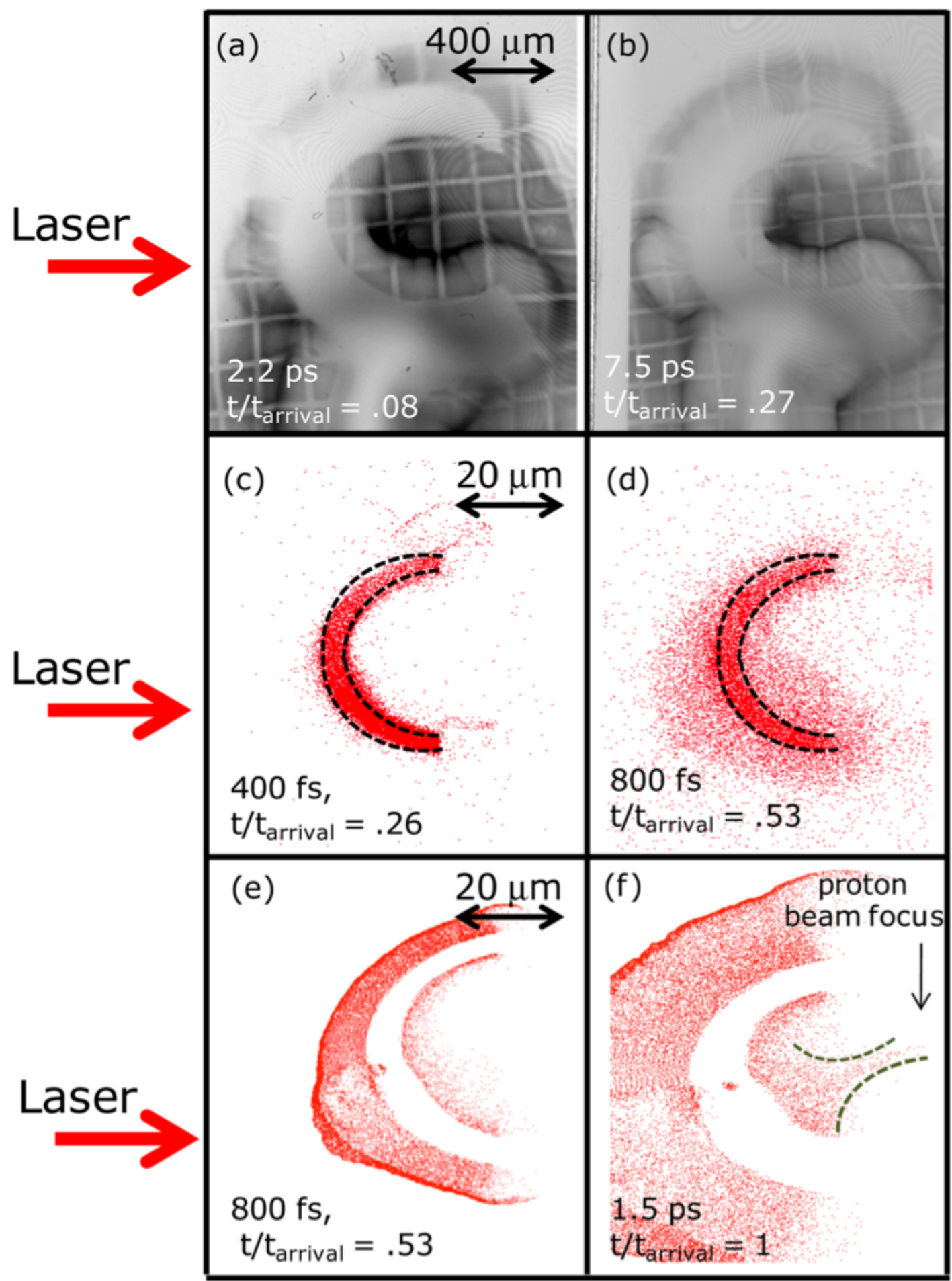

Figure 5: The B1 short pulse laser was aimed off-center on the half cylinder. Deflectograms of the sheath field at $t / t_{\text {arrival }}=0.08$ (a) and 0.27 (b). PICLS simulation results plotting hot electron positions are shown in (c) and (d). Simulated proton positions are shown in (e) and (f) that also show location of the focus. 
In conclusion, we diagnosed converging filaments in the sheath field at the back of a curved, laserirradiated solid foil. The coupling of experimental data and simulation suggests that protons with intermediate energies, which carry most of the beam energy, consistently focus close to the center of the cylinder, even for off-axis laser impact on the half-cylinder. Filamentation in the plasma, however, implies that the proton beam transverse size at focus will be enlarged since filamentation is a manifestation of an emittance degraded beam [30]. All this, resulting here in the observed beam size of $30 \mu \mathrm{m}$, is detrimental to the proton Fast Ignition scheme which would require $10-20 \mu \mathrm{m}$ beam to minimize the required igniter energy [1]. However, simulations shown in Fig.4.c-d suggest that focusing could improve for conditions closer to fast ignition than tested here. The next step will be to test this in future experiments.

We acknowledge the support of the LULI technical teams. This work was supported by DAAD, British Council/Alliance, Grant No. E1127 from Région Ile-de-France, the "ULIMAC" junior chair program of RTRA-Triangle de la Physique, ANR-06-BLAN-0392 from ANR-France, EPSRC, Grants No. EP/C003586/1 and No. EP/E035728/1 (LIBRA consortium), the EU program HPRI-CT-1999-0052, and TR18 and GK1203 funding. E.L acknowledges EURATOM within the "Keep-in-Touch" activities and was granted access to the HPC resources of CINES under the allocation 2011-056129 made by GENCI (Grand Equipement National de Calcul Intensif). S.N.C is supported under the auspices of the U.S. Department of Energy by Lawrence Livermore National Laboratory under Contract \# DE-AC52$07 \mathrm{NA} 27344$. 


\section{REFERENCES}

[1] M. Roth, et al., Phys. Rev. Lett. 86, 436 (2001).

[2] A. Mancic, et al., High Energy Density Physics 6, 21 (2010); A. Pelka et al., Phys. Rev. Lett. 105, 265701 (2010); M. Carrié and E. Lefebvre, High Energy Density Physics 7, 353 (2011).

[3] G. B. Andresen, et al., Phys. Rev. Lett. 98, 023402 (2007); G. B. Andresen, et al., Nature 468, 673 (2010).

[4] S. C. Wilks, et al., Phys. Plasmas 8, 542 (2001).

[5] H. Chen, et al., Phys. Rev. Lett. 105, 015003 (2010).

[6] P. Mora, Phys. Rev. Lett. 90, 185002 (2003).

[7] R. Snavely, et al., Phys. Rev. Lett. 85, 2945 (2000).

[8] J. Fuchs, et al., Phys. Rev. Lett. 91, 255002 (2003).

[9] M. Roth et al., Plasma Phys. Control. Fusion 47, B84 (2005).

[10] T. Toncian, et al., Science 312, 410 (2006)

[11] L. Romagnani, et al., Phys. Rev. Lett. 95, 195001 (2005).

[12] P. Antici et al., Phys. Rev. Lett. 101, 105005 (2008).

[13] P. K. Patel, et al., Phys. Rev. Lett 91, 125004 (2003).

[14] S. Kar et al., Phys. Rev. Lett 106, 225003 (2011).

[15] D.T. Offermann et al., Phys. Plasmas 18, 056713 (2011).

[16] E. Lefebvre, et al., Nucl. Fusion 43, 629 (2003).

[17] Y. Sentoku, and A. J. Kemp, J. Comput. Phys. 227, 6846 (2008).

[18] E. Lefebvre, et al., J. Appl. Phys. 100, 113308 (2006).

[19] M. Borghesi, et al., Plasma Phys. Control. Fusion 50, 124040 (2008).

[20] A. J Mackinnon, et al., Rev. Sci. Inst. 75, 3135 (2004).

[21] M. Borghesi, et al., Appl. Phys. Lett 82, 1529 (2003).

[22] H. Ruhl, et al., Plasma Phys. Reports 27, 363 (2001).

[23] K. Quinn, et al., Eur. Phys. J. D. 55, 293 (2009).

[24] J. Fuchs, et al., Nature Physics 2, 48 (2006).

[25] R. A. Snavely, et al., Physics of Plasmas 14, 092703 (2007).

[26] M. Dunne, Nature Phys. 2, 2 (2006); M. Dunne, HiPER-Technical Background and Conceptual Design Report 2007, Rutherford Appleton Laboratory, Report No. RAL-TR-2007-008 (June 2007), available on the site www.hiper-laser.org.

[27] C. Haefner, et. al., Journal of Physics: Conference Series 244, 032005 (2010). 
[28] M. Key et al., Fusion Sci. and Tech. 49, 440 (2006).

[29] S. Buffechoux, et al., Phys. Rev. Lett. 105, 015005 (2010); T. Toncian et al., Phys. Plasmas 18, 043105 (2011).

[30] A. J. Kemp et al., Phys Rev E 75, 056401 (2007). 